\title{
Ursodeoxycholic acid effectively kills drug-resistant gastric cancer cells through induction of autophagic death
}

\author{
SUNG-CHUL LIM ${ }^{1}$ and SONG IY HAN ${ }^{2}$ \\ ${ }^{1}$ Department of Pathology, Chosun University College of Medicine; ${ }^{2}$ Division of Natural Medical Sciences, \\ College of Health Science, Chosun University, Dong-gu, Gwangju 501-759, Republic of Korea
}

Received April 23, 2015; Accepted May 26, 2015

DOI: $10.3892 /$ or.2015.4076

\begin{abstract}
Carcinoma cells that have acquired drug resistance often exhibit cross-resistance to various other cytotoxic stimuli. Here, we investigated the effects of ursodeoxycholic acid (UDCA), a gastrointestinal tumor-suppressor, on a cisplatin-resistant SNU601 gastric cancer subline (SNU601/R). While other anticancer drugs, including L-OHP, etoposide, and death ligand TRAIL, had minimal effects on the viability of these resistant cells, they were sensitive to UDCA. The UDCA-induced reduction in the viability of the SNU601/R cells was accomplished through autophagy while the primary means of cell death in the parental SNU601 cells (SNU601/WT) was apoptosis. Previously, we demonstrated that the UDCA-triggered apoptosis of gastric cancer cells was regulated by a cell surface death receptor, TRAIL-R2/DR5, which was upregulated and re-distributed on lipid rafts. The UDCA stimulation of TRAIL-R2/DR5 also occurred in the SNU601/R cells despite the lack of apoptosis. In the present study, we found that CD95/Fas, another cell surface death receptor, was also translocated into lipid rafts in response to UDCA although it was not involved in the decrease in cell viability. Specifically, raft relocalization of CD95/Fas was triggered by UDCA in the SNU601/WT cells in which apoptosis occurred, but not in the SNU601/R cells where autophagic death occurred. Notably, UDCA reduced ATG5 levels, an essential component of autophagy, in the SNU601/WT, but not in the SNU601/R cell line. Moreover, in CD95/Fas-silenced SNU601/WT cells, UDCA did not decrease ATG5 levels and induced autophagic cell death rather than apoptosis. These results imply that raft-distributed CD95/Fas may support UDCA-induced apoptosis via downregulation of ATG5 levels, preventing the autophagic pathway. Taken together, these results suggest that UDCA induces both apoptotic and
\end{abstract}

Correspondence to: Professor Song Iy Han, Division of Natural Medical Sciences, College of Health Science, Chosun University, Dong-gu, Gwangju 501-759, Republic of Korea

E-mail: sihan@chosun.ac.kr

Key words: ursodeoxycholic acid, drug-resistance, autophagy, gastric carcinoma cells autophagic cell death depending on the intracellular signaling environment, thereby conferring the advantage to overcome drug resistance through apoptotic defects.

\section{Introduction}

Gastric cancer (GC) is a commonly diagnosed cancer with a low survival rate, resulting in its status as one of the leading causes of cancer-related death. Although surgical tumor removal is highly effective for the treatment of early-stage GC (1), many cases are diagnosed at an advanced stage and cancer recurrence after complete resection is common. Therefore, chemotherapy is an important option for the treatment of unresectable or metastasized cancers. Recently, considerable progress has been achieved in chemotherapeutics, and various cisplatin-based regimens have shown promising anticancer outcomes in GC. However, the use of anticancer drugs can give rise to the drug resistance of cancer cells. In many cases, once cancer cells acquire drug resistance to one type of drug, they tend to show cross-resistance to an array of drugs with different structures and cellular targets. Thus, the acquisition of drug resistance is a serious obstacle to the success of chemotherapy.

The development of drug resistance often results from reduced drug accumulation due to the overexpression of membrane efflux pumps, such as the canalicular multispecific organic anion transporter or P-type adenosine triphosphatase (ATP7B) $(2,3)$. To date, various efforts have been made to reverse drug resistance with inhibitors to these membrane pumps (4), but the inhibitors have not proven clinically useful without side effects. Furthermore, chemotherapeutic drug resistance is a complex phenomenon, which also stems from changes in multiple systems including the upregulation of survival pathways, such as AKT activation and ERK-dependent MKP-1 induction $(2,5,6)$, and the suppression of induced death pathways by defects in the apoptotic pathway or the enhancement of the antioxidant system and the DNA repair pathway $(7,8)$. Therefore, various strategies to overcome resistance by targeting these signaling pathways are being currently evaluated in the field of cancer research.

The hydrophilic bile acid, ursodeoxycholic acid (UDCA), has been regarded as a suppressor of gastrointestinal tumors. In addition, UDCA was implicated in the prevention of colonic cancer through cell cycle arrest and the suppression 
of oncogenic factors including Ras and COX-2 $(9,10)$. In our previous study, we also showed that UDCA performs a suppressive role in tumor progression in gastric carcinoma cells by inducing apoptosis $(11,12)$. However, the role of UDCA in drug-resistant cells is unclear. Our aim in the present study was to understand the underlying mechanisms involved in the effects of UDCA on a cisplatin-resistant sub-cell line of SNU601 gastric cancer cells to aid future progress toward the circumvention of chemoresistance.

\section{Materials and methods}

Cell culture and dosing. The SNU601 human gastric cancer cell line was obtained from the Korea Cell Line Bank, and the cisplatin-resistant SNU601 cells (SNU601/R) were a gift from Professor C.H. Choi (Department of Pharmacology, Chosun University, Korea). The cells were cultured in RPMI-1640 medium (Invitrogen, Carlsbad, CA, USA) supplemented with $10 \%(\mathrm{v} / \mathrm{v})$ fetal bovine serum, and $1 \% \mathrm{PS}$ at $37^{\circ} \mathrm{C}$ in a $5 \% \mathrm{CO}_{2}$ atmosphere. Drug treatment of the cells was performed by adding 500-1000 $\mu \mathrm{M}$ UDCA (ICN Biomedicals, Santa Ana, CA, USA), 10-50 $\mu \mathrm{M}$ oxaliplatin (L-OHP; Boryung Pharmaceutical, Korea), 3-10 $\mu \mathrm{M}$ etoposide (Sigma-Aldrich), or 2-10 $\mathrm{ng} / \mathrm{ml}$ recombinant human TNF-related apoptosisinducing ligand (rhTRAIL), a gift from T.H. Kim (Department of Biochemistry and Molecular Biology, Chosun University, Korea) in the culture medium and incubating it for $48 \mathrm{~h}$. Unless specified, drugs were purchased from Calbiochem (San Diego, CA, USA).

3-(4,5-Dimethylthiazol-2-yl)-2,5-diphenyltetrazolium bromide (MTT) viability assays. For performance of the MTT assay, cells were plated in the wells of a 96-well plate at a density of $1 \times 10^{4}$ cells/well, incubated for $24 \mathrm{~h}$, and then treated with the drugs for $48 \mathrm{~h}$. The MTT solution $(0.5 \mathrm{mg} / \mathrm{ml})$ was added to the wells and incubated at $37^{\circ} \mathrm{C}$ in a $\mathrm{CO}_{2}$ incubator for $4 \mathrm{~h}$. The plates were centrifuged at $600 \mathrm{x} \mathrm{g}$ for $10 \mathrm{~min}$, and the culture medium was removed. The cells were solubilized using dimethyl sulfoxide (DMSO) and the solubilized formazan product was quantified using an enzyme-linked immunosorbent assay (ELISA) plate reader at $595 \mathrm{~nm}$. The absorbance of the untreated cells was designated as $100 \%$, and the cell survival was expressed as a percentage of this value.

Analysis of apoptosis. Treated cells were stained with $1 \mu \mathrm{g} / \mathrm{ml}$ Hoechst 33342 (HO) for $15 \mathrm{~min}$ at room temperature in the dark. Next, both the floating and attached cells were collected and centrifuged. The pooled cell pellets were washed with ice-cold phosphate-buffered saline (PBS), fixed in 3.7\% formaldehyde on ice, washed and resuspended with PBS, and then a fraction of the suspension was centrifuged in a cytospinner (Shandon, Thermo Fisher Scientific, Waltham, MA, USA). Slides were prepared, air dried, mounted in anti-fade solution, and observed under a fluorescence microscope (DM5000; Leica, Washington, NY, USA) as described elsewhere. Any condensed/fragmented nuclei were assessed as apoptotic cells. A total of 500 cells distributed across random microscopic viewing fields were counted, and the number of apoptotic cells was expressed as a percentage of the total number of cells counted.
Immunoblotting. Equal amounts of protein extracts were electrophoretically separated using 10-12\% SDS-PAGE and transferred to a nitrocellulose membrane using a standard technique. Antibodies were used to probe for cleaved cysteinyl aspartate-specific protease-3 (caspase-3; Cell Signaling Technology, Danvers, MA, USA); poly(ADP-ribose) polymerase (PARP), CD95/Fas, FADD, cytochrome $c$, caveolin-1 (Santa Cruz Biotechnology, Santa Cruz, CA, USA); TRAIL-R1/DR4, TRAIL-R2/DR5 (ProSci, Poway, CA, USA); ATG5, LC3II (MBL International, Woburn, MA, USA); and c-FLIP (Alexis Biochemicals, Farmingdale, NY, USA). Anti- $\alpha$-tubulin (BioGenex, San Ramon, CA, USA) was used as a loading control. Signals were acquired using an Image Station 4000MM image analyzer (Kodak, Rochester, NY, USA).

Caspase- 8 activity assays. Caspase- 8 activity was assayed using an FADD-like IL-1 $\beta$-converting enzyme (FLICE) colorimetric assay kit (BioVision, Inc., Milpitas, CA, USA), according to the manufacturer's protocol. Briefly, $200 \mu \mathrm{g}$ of protein extract in a $50 \mu \mathrm{l}$ volume was mixed with the reaction buffer and the IETD-pNA substrate, incubated for $90 \mathrm{~min}$, and the absorbance at $405 \mathrm{~nm}$ was measured. The fold increase in FLICE activity was determined from comparison of the results of the treated samples with the untreated control.

RNA interference (RNAi). For the RNAi experiment, siRNA of TRAILR-1/DR4, 5'-CUGGAAAGUUCAUCUACUU(dtdt)-3' (sense) and 5'-AAGUAGAUGAACUUUCCAG(dtdt)-3' (antisense); TRAILR-2/DR5, 5'-CAGACUUGGUGCCCUUUG (dtdt)-3' (sense) and 5'-UCAAAGGGCACCAAGUCUG (dtdt)-3' (antisense); CD95/Fas, 5'-GAGAGUAUUACUA GAG CUU(dtdt)-3' (sense) and 5'-AAGCUCUAGUAAUACU CUC(dtdt)-3' (antisense); and 5'-control siRNA, 5'-CCUACG CCACCAAUUUCGU(dtdt)-3' (sense) and 5'-ACGAAAUUG GUGGCGUAGG(dtdt)-3' (antisense) were purchased from Bioneer (Daejeon, Korea). Cells were individually transfected with siRNA oligonucleotides using an Amaxa Transfection system $^{\mathrm{TM}}$ (Basel, Switzerland) and grown for $24 \mathrm{~h}$ prior to drug treatment.

Lipid raft fractionation. Lipid rafts were isolated by sucrose density-gradient centrifugation. A total of $10^{8}$ cells were lysed for $30 \mathrm{~min}$ in $1 \mathrm{ml}$ of lysis buffer (1\% Brij35 in HEPES buffer; $25 \mathrm{mM}$ HEPES, $1 \mathrm{mM}$ EDTA, and $150 \mathrm{mM} \mathrm{NaCl}, \mathrm{pH}$ 6.5) supplemented with a protease inhibitor cocktail, followed by homogenization with a glass dounce homogenizer. The homogenates were mixed with $1 \mathrm{ml}$ of $80 \%$ sucrose in HEPES buffer and placed at the bottom of a centrifuge tube. The samples were then overlaid with $6.5 \mathrm{ml}$ of $30 \%$ sucrose followed by $3 \mathrm{ml}$ of $5 \%$ sucrose and centrifuged at $188,000 \mathrm{x} \mathrm{g}$ for $18 \mathrm{~h}$ at $4^{\circ} \mathrm{C}$. Fractions $(1 \mathrm{ml})$ were collected from the bottom to the top of the gradient, and rafts were determined by measurement of the total cholesterol using a cholesterol assay kit (Wako Diagnostics, Richmond, VA, USA). Fractions 3 through 5 of the sucrose gradients were pooled and used as the raft fraction; the rest was designated as the non-raft fraction.

Statistical analysis. All numerical data are reported as mean $\pm \mathrm{SE}$. All data represent the results of at least 3 inde- 
A
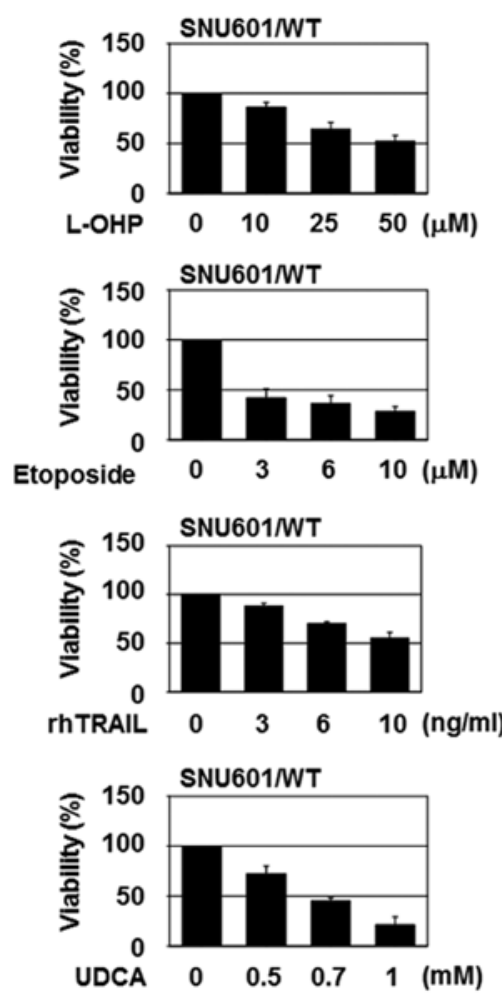
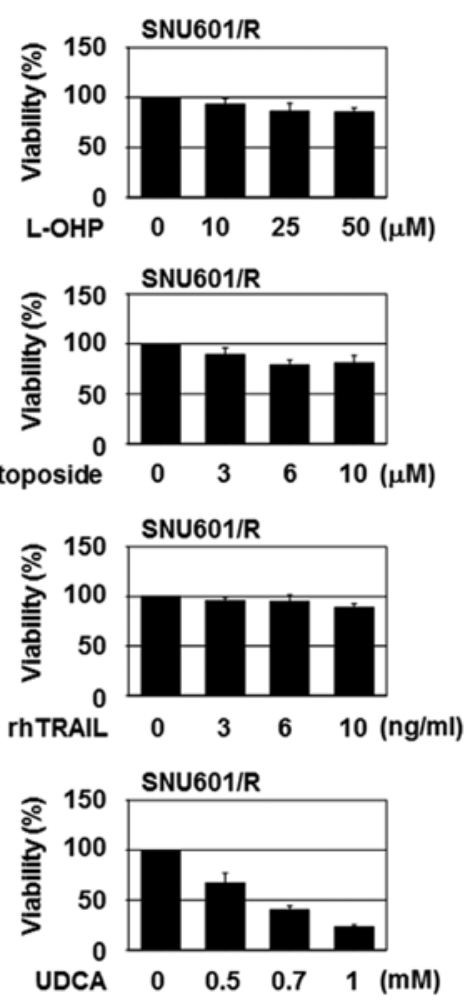

B

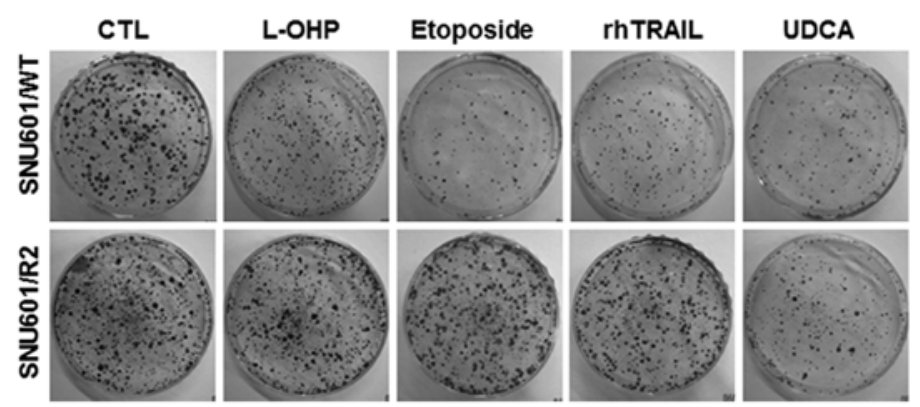

Figure 1. UDCA reduces the viability of a cisplatin-resistant gastric cancer cell line. (A) SNU601/WT and SNU601/R cells were exposed to the indicated concentrations of L-OHP, etoposide, rhTRAIL and UDCA for $48 \mathrm{~h}$, and cell viability was monitored with MTT assay. (B) SNU601/WT and SNU601/R cells exposed to $50 \mu \mathrm{M} \mathrm{L-OHP,} 10 \mu \mathrm{M}$ etoposide, $10 \mathrm{ng} / \mathrm{ml}$ rhTRAIL and $0.7 \mathrm{mM}$ UDCA for $24 \mathrm{~h}$ were trypsinized, and 2,000 cells were re-seeded and cultured for 2 weeks in a $37^{\circ} \mathrm{C}, 5 \% \mathrm{CO}_{2}$ incubator, and colonies were visualized by crystal violet staining. UDCA, ursodeoxycholic acid; L-OHP, oxaliplatin; rhTRAIL, recombinant human TNF-related apoptosis-inducing ligand.

pendent experiments. Groups were compared by means of a Student's t-test.

\section{Results}

UDCA reduces the viability of the cisplatin-resistant variant of the gastric cancer cell lines. Cisplatin-resistant human cancer cells have been reported to have a cross-resistance to various cytotoxic stimuli $(13,14)$. In agreement with this, the cisplatinresistant variant (SNU601/R) derived from the SNU601 human gastric cancer cell line (SNU601/WT) demonstrated a strong resistance to a wide array of anticancer drugs not only to cisplatin (15). In this study, we used SNU601/R cells to evaluate the efficacy of several different types of anticancer drugs, and found that sensitivity to UDCA reduced the cell viability, similar to the parental SNU601/WT cells. However, the other anticancer drugs tested (L-OHP, etoposide and rhTRAIL) had minimal effects on the viability of the resistant cells in doses that led to $\sim 50 \%$ death in the parental cells (Fig. 1A). In addition, only treatment with UDCA decreased the colony-forming ability of the SNU601/R cells (Fig. 1B).

UDCA induces autophagic death in the SNU601/R cells. As UDCA was the only cytotoxic agent tested to reduce the viability of the SNU601/R cells, we explored whether the mechanism involved apoptosis since UDCA was found to induce apoptosis in the SNU601/WT cells in our previous study (11). Both the parental and the resistant cells were exposed to L-OHP, etoposide, rhTRAIL and UDCA, and the resulting apoptotic nuclei were detected by $\mathrm{HO}$ staining. Although UDCA decreased the cell viability of the SNU601/R cells, UDCA-induced apoptotic body formation was very low in comparison to the parental cells (Fig. 2A and B). Other characteristic apoptotic features, namely, the cleavage of 
A
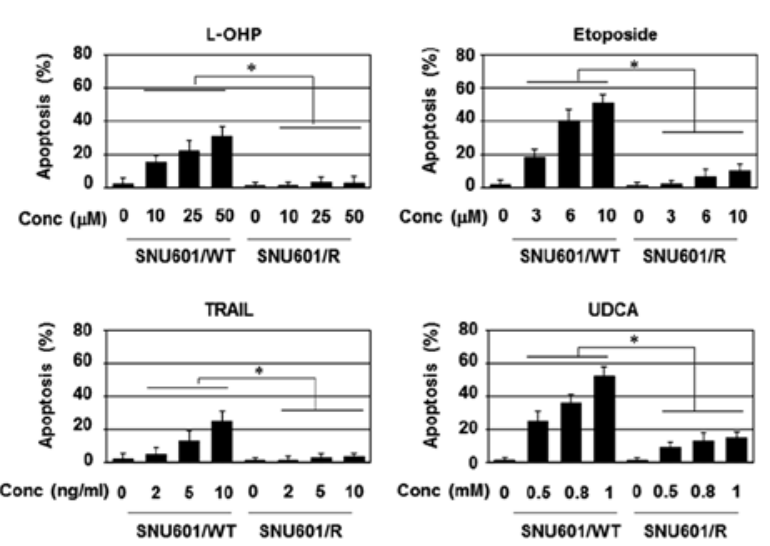

Conc $(\mathrm{mM}) \quad 0 \quad 0.5 \quad 0.8 \quad 1 \quad 00.5 \quad 0.8 \quad 1$ $\overline{\text { SNU601/WT }} \overline{\text { SNU601/R }}$
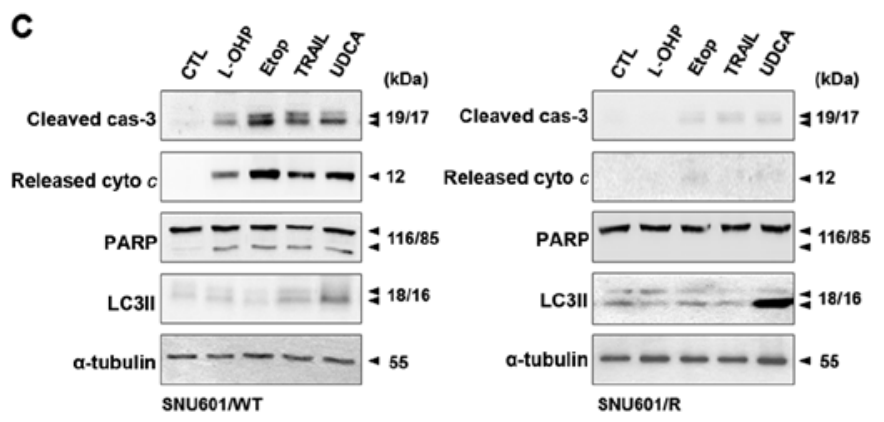

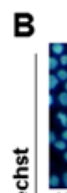

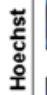
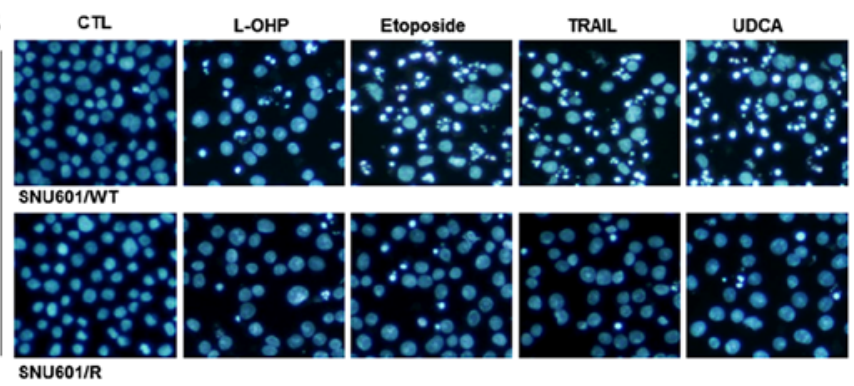

$\mathrm{cT}$
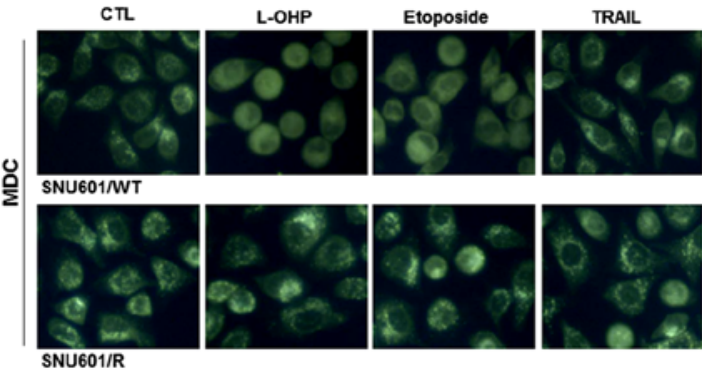

UDCA
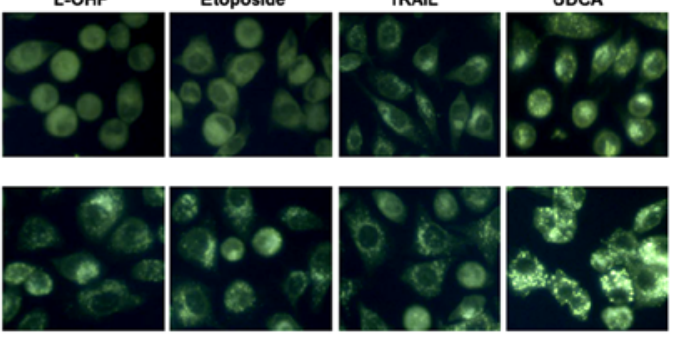

Figure 2. UDCA induces autophagy instead of apoptosis in the cisplatin-resistant variant of SNU601 cells. (A) SNU601/WT and SNU601/R cells were exposed to the indicated doses of L-OHP, etoposide, rhTRAIL and UDCA for $48 \mathrm{~h}$, and were stained by HO. Apoptotic nuclei were counted under a fluorescence microscope, and the number of apoptotic cells was expressed as a percentage of the total number of cells counted. " $<<0.01$. (B and C) SNU601/WT and SNU601/R cells were exposed to vehicle, L-OHP, etoposide, rhTRAIL and UDCA. (B) The treated cells were stained with HO (upper) and MDC (lower) to visualize apoptotic cells and autophagosomes, respectively. Stained cells were observed under a fluorescence microscope (magnification, $\mathrm{x} 200 \mathrm{for} \mathrm{HO}$ image and $\mathrm{x} 400$ for MDC image), or (C) analyzed by immunoblotting to detect cleaved caspase, released cytoplasmic cytochrome $c$, PARP and LC3II. $\alpha$-tubulin was used as a loading control. UDCA, ursodeoxycholic acid; L-OHP, oxaliplatin; rhTRAIL, recombinant human TNF-related apoptosis-inducing ligand; HO, Hoechst 33342; MDC, monodansylcadaverine; PARP, poly(ADP-ribose) polymerase.

caspase-3, the release of cytochrome $c$, and the degradation of PARP, were also blocked in the resistant cells (Fig. 2C). These results indicated that UDCA did not induce apoptosis in the SNU601/R cells.

Thus, we examined whether other types of cell death are involved in UDCA-induced cytotoxicity. Upon treatment with UDCA, the number of autophagic vacuoles was greatly increased in the SNU601/R cells, as visualized by monodansylcadaverine (MDC) and HO staining (Fig. 2B). Moreover, treatment with UDCA resulted in a strong elevation in the level of protein LC3II, an indication of autophagy in the SNU601/R cells (Fig. 2C). These results suggest that autophagy occurred in the resistant cells in response to UDCA.

Autophagy may function as a protective mechanism and as a cell death mechanism depending on the genetic or signaling environments. In this study, inhibition of autophagy by 3-methyladenine (3-MA) did not increase UDCA-induced cell toxicity (data not shown), implying that UDCA-triggered autophagy was not protective autophagy, but a process of cell death. In contrast, necrotic features, PI inclusion, or LDH release were not detected upon UDCA treatment in the SNU601/R cells after $72 \mathrm{~h}$ (data not shown). Therefore, UDCA appears to trigger cell death by autophagy in SNU601/R cells.

$c$-FLIP levels are elevated in the SNU601/R cells. The apoptotic role of TRAIL-R2/DR5 in UDCA-treated parental
SNU601 cells was confirmed via knockdown assays with TRAIL-R2/DR5-targeting siRNA, resulting in the profound suppression of caspase-3 activation and formation of apoptotic nuclei (Fig. 3A). In the SNU601/R cells, UDCA-induced activation of caspase-8, as well as caspase-3, was severely suppressed compared to the parental cells (Fig. 3B). Thus, we aimed to ascertain whether the expression or regulation of the components of DISC was altered in the SNU601/R cells, since DISC is directly linked to caspase- 8 activation.

First, we determined the protein levels of TRAIL-R2/DR5 and the adaptor protein, FADD, in the resistant cells with immunoblotting. The basal levels of TRAIL-R2/DR5 and FADD did not differ between the resistant cells and the parental cells, and an elevation in the expression of TRAIL-R2/DR5 in the UDCA-treated cells was also detected in the resistant cells (Fig. 3C).

We also examined protein levels of other death receptors, including TRAIL-R1/DR4 and CD95/Fas, and DISC inhibitory factor, c-FLIP, to exclude their possible involvement in the activation of UDCA-mediated DISC complex. The basal or UDCA-exposed protein levels of CD95/Fas and TRAIL-R1/DR4 were not altered in the resistant cells (Fig. 3C). However, the expression of the long splice variant of cellular FLICE-inhibitory proteins [c-FLIP(L)] was enhanced in the SNU601/R cells (Fig. 3C). c-FLIP is a death-effector domain (DED)-containing protein that is recruited to DISC and inter- 
A
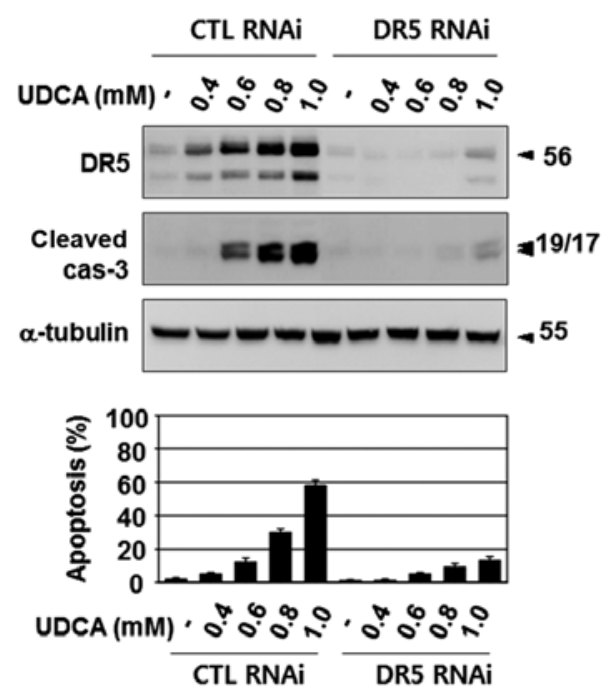

B
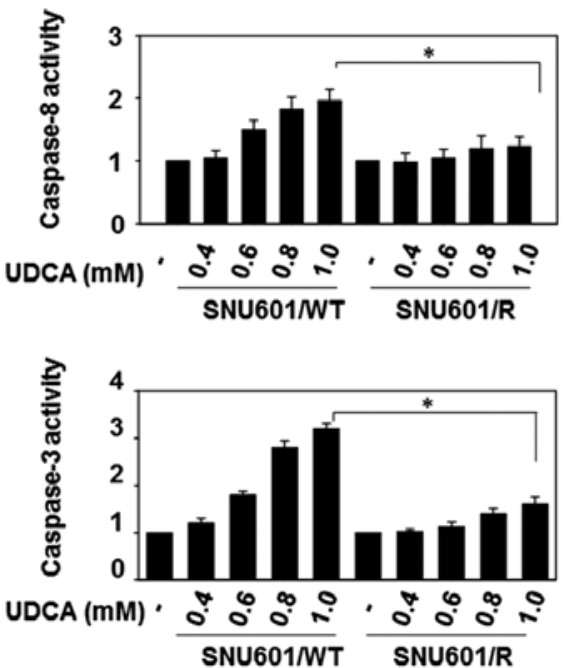

C

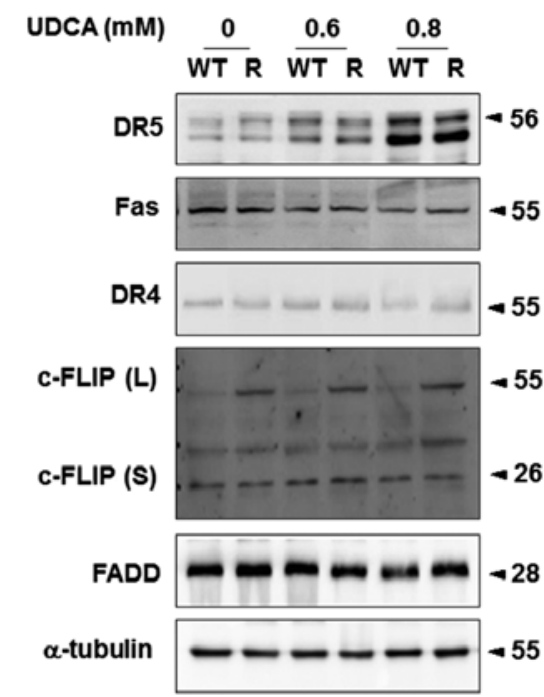

Figure 3. c-FLIP(L) is overexpressed and UDCA-induced caspase-8 activation is repressed in the SNU601/R cells. (A) SNU601/WT cells were transfected with scrambled, control RNA or TRAIL-R2/DR5 siRNA, exposed to UDCA, and analyzed by immunoblotting and apoptotic body staining using HO. (B) SNU601/WT and SNU601/R cells were exposed to the indicated doses of UDCA and subjected to caspase-8 and caspase-3 activity assays. "p $<0.01$. (C) SNU601/WT and SNU601/R cells treated with UDCA were analyzed by immunoblotting to detect TRAIL-R2/DR5, TRAIL-R1/DR4, CD95/Fas, c-FLIP and FADD. UDCA, ursodeoxycholic acid; HO, Hoechst 33342.

feres with caspase-8 activation in death receptor signaling. Therefore, reduction in apoptosis in the resistant cells in response to various apoptotic stimuli may be due to an increase in c-FLIP(L) expression, which inhibits DISC activation.

CD95/Fas is involved in the determination of UDCA-induced cell death mode. Although the elevated expression of c-FLIP may inhibit TRAIL-R2/DR5-mediated apoptotic signaling, the mechanism by which autophagic death is triggered by UDCA in the resistant cells remained unclear. Autophagy is an intracellular degradation system often triggered by nutrient or energy limitation, which is regulated by the Atg protein family. Unexpectedly, we found that the membrane death receptor, CD95/Fas, is associated with the decision of the mode of cell death in the presence of UDCA, despite the fact that TRAIL-R2/DR5 acts as a regulator of UDCA-mediated cell death.

The protein levels of CD95/Fas were observed via immunoblotting, in the presence and absence of UDCA, and the expression levels were similar in both cell lines under both conditions (Figs. 3C and 4A). However, in a raft isolation study, we found that UDCA increased raft-localized CD95/Fas in the SNU601/WT cells, but the raft relocalization was not observed in the SNU601/R cells (Fig. 4B). Translocation of death receptors into raft areas is one of the essential regulatory processes of their activity. Therefore, this result implies that CD95/Fas plays a role in UDCA-treated SNU601/WT, but not in SNU601/R cells.

To understand the role of CD95/Fas further, we performed interference studies in the parental cells. As shown in Fig. 4C, silencing of TRAIL-R2/DR5 simply blocked UDCA-induced apoptosis, as detected by the decrease in cleaved caspase- 3 and the release of cytochrome $c$. Nevertheless, knockdown of CD95/Fas switched the UDCA-induced death pattern from apoptosis to autophagy. This was evidenced by a decrease in apoptotic features, including caspase- 3 cleavage, cytochrome $c$ release, apoptotic nuclei, and the presence of autophagic markers such as LC3II and autophagosomes (Fig. 4C and D). However, knockdown of CD95/Fas did not recover cell viability despite suppression of apoptosis (Fig. 4E), and prevention of autophagy by 3-MA did not further increase the cell death rate under the CD95/Fas-silenced condition (data not shown). Hence, the observed autophagy in the absence of CD95/Fas was also regarded as a type of cell death, not a protective autophagy, as observed in the SNU601/R cells.

Finally, we explored whether essential autophagy regulatory proteins, ATG5 and beclin 1, are involved in the process of the observed cell death. Expression levels of beclin 1 were unchanged in response to UDCA, in control or CD95/Fas-silenced cells; however, ATG5 protein levels were diminished by the presence of UDCA in the control cells, but not in the CD95/Fas-silenced cells (Fig. 4F). Furthermore, UDCA-triggered downregulation of ATG5 was protected in the SNU601/R cells (Fig. 4G). Taken together, these results indicate that CD95/Fas assists TRAIL-R2/DR5 in the induction of apoptosis by inhibiting the autophagic pathway via downregulation of ATG5, and the loss of CD95/Fas activity results in the release of autophagic pathway. Therefore, UDCA appears to induce both apoptosis and autophagic death depending on the intracellular signaling environment.

\section{Discussion}

Apoptosis is a type of programmed cell-suicide in response to various cytotoxic stimuli, such as anticancer drugs, and is typically characterized by membrane blebbing, DNA fragmentation, and apoptotic nuclei. The central components of the apoptotic machinery are caspases $(16,17)$, and the caspase 
A

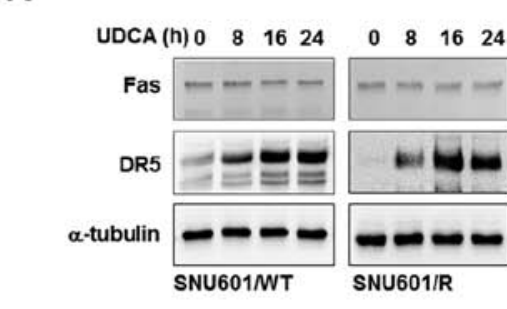

C

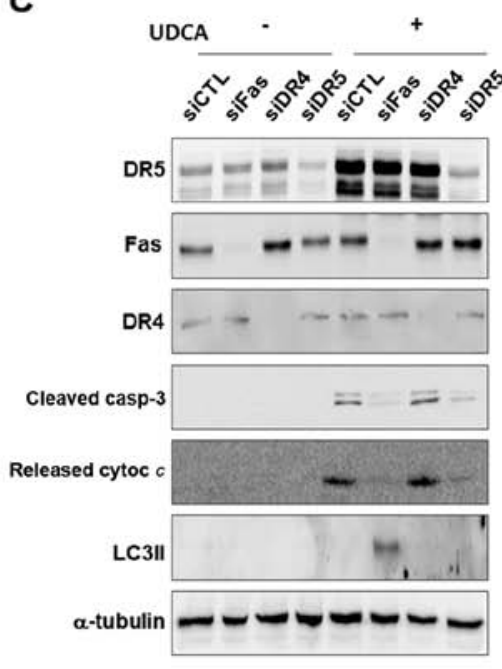

B

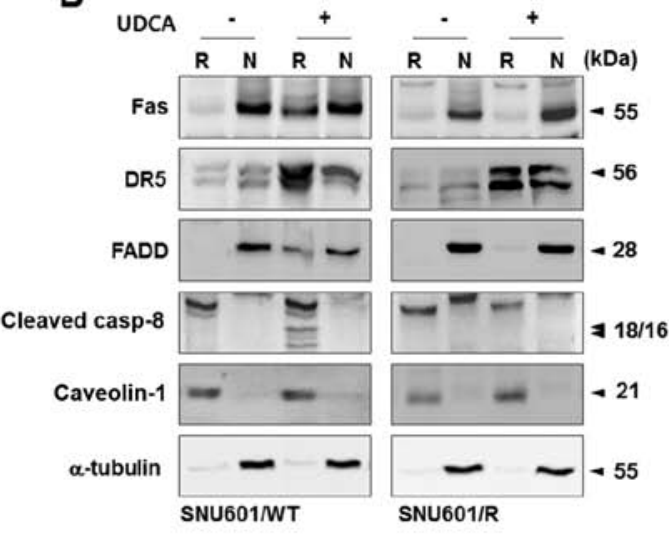

D

UDCA

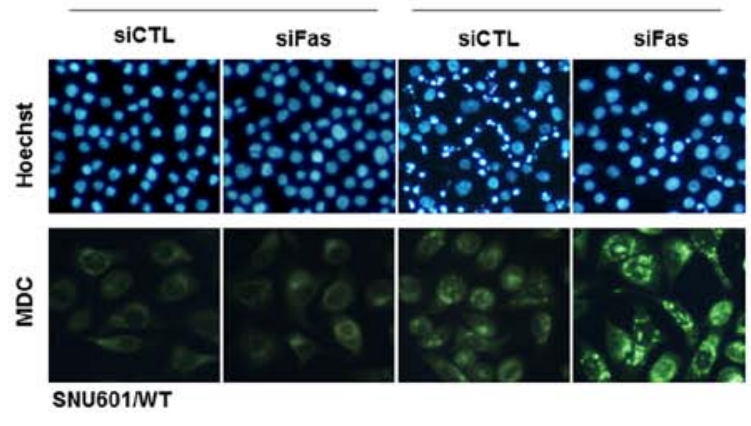

$\mathbf{E}$

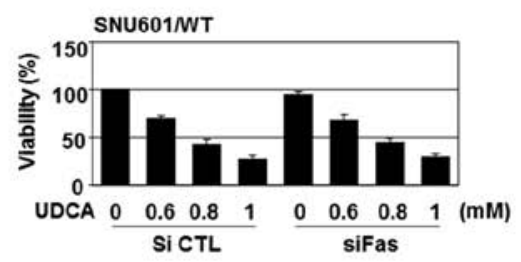

$\mathbf{F}$

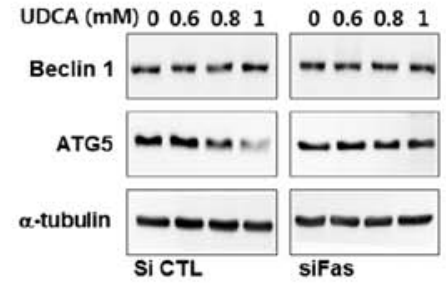

G

$\operatorname{UDCA}(\mathrm{mM}) 00.60 .81$

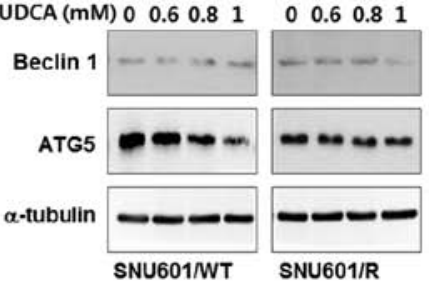

Figure 4. Raft localization of CD95/Fas appears to be involved in the type of UDCA-induced cell death. (A) SNU601/WT and SNU601/R cells were treated with UDCA for the indicated times and analyzed by immunoblotting to detect TRAIL-R2/DR5 and CD95/Fas. (B) SNU601/WT and SNU601/R cells were incubated in the absence or presence of UDCA, separated into raft $(\mathrm{R})$ and non-raft $(\mathrm{N})$ fractions, and analyzed by immunoblotting using antibodies against CD95/Fas, TRAIL-R2/DR5, FADD, caspase-8, caveolin-1 and $\alpha$-tubulin. (C) SNU601/WT cells, transfected with scrambled control RNA or siRNAs for CD95/Fas, TRAIL-R1/DR4, and TRAIL-R2/DR5, were exposed to the vehicle control or UDCA and subjected to immunoblotting. (D-F) SNU601/WT cells, transfected with control scrambled RNA or CD95/Fas siRNA were exposed to vehicle or UDCA, and analyzed by (D) HO or MDC staining, (E) MTT assay, and (F) immunoblotting using antibodies against beclin1 and ATG5. (G) SNU601/WT and SNU601/R cells were incubated with UDCA and analyzed by immunoblotting to detect beclin1 and ATG5. UDCA, ursodeoxycholic acid; HO, Hoechst 33342; MDC, monodansylcadaverine.

cascade is signaled by two main pathways. The first is an extrinsic pathway, which is initiated by ligation of cell surface death receptors, leading to DISC formation and caspase- 8 activation. The second is an intrinsic mitochondrial pathway, triggered by cytochrome $c$ release from the mitochondria, and subsequent caspase-9 activation (16-18). These extrinsic and intrinsic pathways are often linked and they work cooperatively to promote apoptosis. Together caspase- 8 and -9 activate caspase- 3 , the main executioner of the apoptotic pathway that leads to cleavage of cellular proteins, such as poly(ADP-ribose) polymerase $(16,18)$. However, cancer cells that survive cytotoxic stimuli often have defects in apoptosis from the alteration of signaling molecules in the extrinsic and intrinsic pathways, such as surface death receptors, p53 and Bcl-2 family members (19-21). Likewise, the signaling defects in the main apoptotic pathway cause cross-resistance to various anticancer agents that act through this pathway.

In this case, stimulation of other types of cell death rather than apoptosis could be a strategy to circumvent apoptotic aberrations. To date, necrosis and autophagic death have been identified as distinct types of cell death. Necrosis is a strong cellular destructive process; however, induction of necrosis for cancer therapeutics requires a careful approach as a necrosisinduced massive inflammatory response promotes tumor growth or angiogenesis by releasing intracellular cytokines, such as HMGB1 (22-27).

Autophagy is another type of self-degradative process involved in the basal turnover of cellular components in response to nutrient starvation or organelle damage, and it is controlled by autophagy-related ATG proteins, including beclin 1 and ATG5. This process is characterized by sequestration of intracellular organelles or portions of the cytoplasm in autophagosomes, which are degraded after fusion with lysosomes for subsequent recycling. This process primarily acts as a pro-survival mechanism to overcome a stressful condition, but it ultimately commits the cell to death under prolonged or severely stressful environments (28). This autophagic death is suggested to be a form of tumor-suppressive cell death, as shown by the use of tumor-suppressors such as beclin 1 and DAP-kinase in autophagic pathways. Therefore, autophagic death is expected to be a suitable option for therapeutic treatment of cancers that harbor defects in apoptotic signaling.

In this study, we showed that cisplatin-resistant cancer cells were insensitive to various anticancer agents including a platinum-based drug (L-OHP), a topoisomerase inhibitor (etoposide) and a specific ligand to surface death receptor (rhTRAIL). Thus, SNU601/R cells may have defects in their apoptotic machinery. However, UDCA was able to reduce the 
cell viability and markedly elevate autophagic death, but not apoptosis, in the drug-resistant cells. These results suggest that autophagic death is responsible for the decrease in viability in the resistant cells, despite the fact that UDCA acted as an apoptotic inducer in the parental, non-resistant cells.

Next, we investigated how UDCA could induce autophagic death in drug-resistant cells. Previously, we showed that UDCA elevated the protein levels of TRAIL-R2/DR5, which plays a critical role in UDCA-induced apoptosis; however, TRAIL-R2/DR5-mediated apoptosis requires the activity of CD95/Fas. In the parental cells, translocation of CD95/Fas into membrane rafts was detected in the UDCA-treated cells, although CD95/Fas levels were not increased. In addition, knockdown of CD95/Fas in the parental cells significantly blocked UDCA-induced apoptotic characteristics, such as caspase cleavage and cytochrome $c$ release, but, in contrast, encouraged autophagic features, including higher LC3II levels and the presence of autophagic vacuoles. Furthermore, UDCA treatment decreased ATG5 levels in the parental cells, whereas the silencing of CD95/Fas protected these protein levels. Nevertheless, the knockdown of CD95/Fas did not affect the cell viability, while knockdown of TRAIL-R2/DR5 partially restored cell viability, in the UDCA-treated cells. These results suggest that CD95/Fas contributes to the induction of apoptosis by blocking autophagic processes via inhibition of ATG5 protein levels. In this context, the absence of CD95/Fas redistribution in the resistant cells appears to be linked to a release in autophagic signaling, which results in autophagic death. In support of our hypothesis, ATG5 levels were not reduced in the UDCA-treated resistant cells. We also found that the resistant cells had an elevated level of c-FLIP(L), indicating that the repression of DISC activity is one reason for the multi-drug resistance of the resistant cells. Therefore, stimulation of autophagic signaling, which does not require DISC formation, could be an efficient bypass mechanism to overcome the resistance.

Our results revealed that at least two surface death receptors, TRAIL-R2/DR5 and CD95/Fas, were stimulated by UDCA, and cooperation of different death receptors can modulate the mode of cell death, thereby taking advantage of the intracellular signaling environment to stimulate various types of death. In fact, several studies have suggested that several molecular nodes of crosstalk interconnect autophagy and apoptosis, and autophagy can be triggered as an evasive system to avoid apoptosis $(29,30)$. Although further studies are required to clarify the exact mechanism by which UDCA-mediated CD95/Fas signaling is regulated, our results demonstrated that UDCA can stimulate both apoptotic and autophagic death, and may provide at least a part of the explanation of why the resistant cells are sensitive to UDCA. This finding may provide a possible strategy to improve chemotherapeutic efficacy in apoptosis-inducing drug-resistant cancers.

\section{Acknowledgements}

This research was supported by the Basic Science Research Program through the National Research Foundation of Korea (NRF) funded by the Ministry of Education, Science and Technology (NRF-2011-0014540), and through the Research Center for Resistant Cells (R13-2003-009). We thank
Professor Cheol-Hee Choi for the cisplatin-resistant SNU601 cells, Professor Tae-Hyoung Kim for the kind gift of rhTRAIL, and Ms. Jeong-Eun Choi and Dr Hong-Quan Duong for their excellent technical assistance.

\section{References}

1. Brozovic A, Fritz G, Christmann M, Zisowsky J, Jaehde U, Osmak M and Kaina B: Long-term activation of SAPK/JNK, p38 kinase and fas-L expression by cisplatin is attenuated in human carcinoma cells that acquired drug resistance. Int J Cancer 112: 974-985, 2004.

2. Komatsu M, Sumizawa T, Mutoh M, Chen ZS, Terada K, Furukawa T, Yang XL, Gao H, Miura N, Sugiyama T, et al: Copper-transporting P-type adenosine triphosphatase (ATP7B) is associated with cisplatin resistance. Cancer Res 60: 1312-1316, 2000.

3. Taniguchi K, Wada M, Kohno K, Nakamura T, Kawabe T, Kawakami M, Kagotani K, Okumura K, Akiyama S and Kuwano M: A human canalicular multispecific organic anion transporter (cMOAT) gene is overexpressed in cisplatin-resistant human cancer cell lines with decreased drug accumulation. Cancer Res 56: 4124-4129, 1996.

4. Zhu HJ, Wang JS, Guo QL, Jiang Y and Liu GQ: Reversal of $\mathrm{P}$-glycoprotein mediated multidrug resistance in K562 cell line by a novel synthetic calmodulin inhibitor, E6. Biol Pharm Bull 28: 1974-1978, 2005.

5. McCubrey JA, Steelman LS, Abrams SL, Lee JT, Chang F, Bertrand FE, Navolanic PM, Terrian DM, Franklin RA, D'Assoro AB, et al: Roles of the RAF/MEK/ERK and PI3K/PTEN/AKT pathways in malignant transformation and drug resistance. Adv Enzyme Regul 46: 249-279, 2006.

6. Wang J, Zhou JY and Wu GS: ERK-dependent MKP-1-mediated cisplatin resistance in human ovarian cancer cells. Cancer Res 67: 11933-11941, 2007.

7. Choi CH, Kim HS, Kweon OS, Lee TB, You HJ, Rha HS, Jeong JH, Lim DY, Min YD, Kim MS, et al: Reactive oxygen species-specific mechanisms of drug resistance in paraquat-resistant acute myelogenous leukemia sublines. Mol Cells 10: 38-46, 2000.

8. Xu Z, Chen ZP, Malapetsa A, Alaoui-Jamali M, Bergeron J, Monks A, Myers TG, Mohr G, Sausville EA, Scudiero DA, et al: DNA repair protein levels vis-à-vis anticancer drug resistance in the human tumor cell lines of the National Cancer Institute drug screening program. Anticancer Drugs 13: 511-519, 2002.

9. Alberts DS, Martínez ME, Hess LM, Einspahr JG, Green SB, Bhattacharyya AK, Guillen J, Krutzsch M, Batta AK, Salen G, et al; Phoenix and Tucson Gastroenterologist Networks: Phase III trial of ursodeoxycholic acid to prevent colorectal adenoma recurrence. J Natl Cancer Inst 97: 846-853, 2005.

10. Khare S, Cerda S, Wali RK, von Lintig FC, Tretiakova M, Joseph L, Stoiber D, Cohen G, Nimmagadda K, Hart J, et al: Ursodeoxycholic acid inhibits Ras mutations, wild-type Ras activation, and cyclooxygenase- 2 expression in colon cancer. Cancer Res 63: 3517-3523, 2003.

11. Lim SC, Duong HQ, Choi JE, Lee TB, Kang JH, Oh SH and Han SI: Lipid raft-dependent death receptor 5 (DR5) expression and activation are critical for ursodeoxycholic acid-induced apoptosis in gastric cancer cells. Carcinogenesis 32: 723-731, 2011.

12. Lim SC, Duong HQ, Parajuli KR and Han SI: Pro-apoptotic role of the MEK/ERK pathway in ursodeoxycholic acid-induced apoptosis in SNU601 gastric cancer cells. Oncol Rep 28: 1429-1434, 2012.

13. Iwasaki I, Sugiyama H,Kanazawa S and Hemmi H: Establishment of cisplatin-resistant variants of human neuroblastoma cell lines, TGW and GOTO, and their drug cross-resistance profiles. Cancer Chemother Pharmacol 49: 438-444, 2002.

14. Sun CL and Chao CC: Cross-resistance to death ligand-induced apoptosis in cisplatin-selected HeLa cells associated with overexpression of DDB2 and subsequent induction of cFLIP. Mol Pharmacol 67: 1307-1314, 2005.

15. Xu H, Choi SM, An CS, Min YD, Kim KC, Kim KJ and Choi $\mathrm{CH}$ : Concentration-dependent collateral sensitivity of cisplatin-resistant gastric cancer cell sublines. Biochem Biophys Res Commun 328: 618-622, 2005.

16. Hengartner MO: The biochemistry of apoptosis. Nature 407: $770-776,2000$ 
17. Martinou JC and Green DR: Breaking the mitochondrial barrier. Nat Rev Mol Cell Biol 2: 63-67, 2001.

18. Tsujimoto Y: Cell death regulation by the Bcl-2 protein family in the mitochondria. J Cell Physiol 195: 158-167, 2003.

19. Zeuner A, Pedini F, Signore M, Ruscio G, Messina C, Tafuri A, Girelli G, Peschle C and De Maria R: Increased death receptor

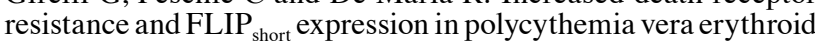
precursor cells. Blood 107: 3495-3502, 2006.

20. Müllauer L, Gruber P, Sebinger D, Buch J, Wohlfart S and Chott A: Mutations in apoptosis genes: A pathogenetic factor for human disease. Mutat Res 488: 211-231, 2001.

21. Perego P, Giarola M, Righetti SC, Supino R, Caserini C, Delia D, Pierotti MA, Miyashita T, Reed JC and Zunino F: Association between cisplatin resistance and mutation of p53 gene and reduced bax expression in ovarian carcinoma cell systems. Cancer Res 56: 556-562, 1996.

22. Degenhardt K, Mathew R, Beaudoin B, Bray K, Anderson D, Chen G, Mukherjee C, Shi Y, Gélinas C, Fan Y, et al: Autophagy promotes tumor cell survival and restricts necrosis, inflammation, and tumorigenesis. Cancer Cell 10: 51-64, 2006.
23. Hippert MM, O'Toole PS and Thorburn A: Autophagy in cancer: Good, bad, or both? Cancer Res 66: 9349-9351, 2006.

24. Liang XH, Jackson S, Seaman M, Brown K, Kempkes B, Hibshoosh $\mathrm{H}$ and Levine B: Induction of autophagy and inhibition of tumorigenesis by beclin 1. Nature 402: 672-676, 1999.

25. Syntichaki P and Tavernarakis N: The biochemistry of neuronal necrosis: Rogue biology? Nat Rev Neurosci 4: 672-684, 2003.

26. Vakkila $\mathbf{J}$ and Lotze MT: Inflammation and necrosis promote tumour growth. Nat Rev Immunol 4: 641-648, 2004.

27. Zong WX and Thompson CB: Necrotic death as a cell fate. Genes Dev 20: 1-15, 2006.

28. Bernales S, McDonald KL and Walter P: Autophagy counterbalances endoplasmic reticulum expansion during the unfolded protein response. PLoS Biol 4: e423, 2006.

29. Eisenberg-Lerner A, Bialik S, Simon HU and Kimchi A: Life and death partners: Apoptosis, autophagy and the cross-talk between them. Cell Death Differ 16: 966-975, 2009.

30. Moretti L, Cha YI, Niermann KJ and Lu B: Switch between apoptosis and autophagy: Radiation-induced endoplasmic reticulum stress? Cell Cycle 6: 793-798, 2007. 